FOLIA

Amazónica

Revista del Instituto de Investigaciones

de la Amazonía Peruana

\title{
MURCIÉLAGOS INDICADORES DE HÁBITATS PERTURBADOS EN LA RESERVA NACIONAL ALLPAHUAYO MISHANA, AMAZONÍA PERUANA
}

\author{
María Claudia RAMOS-RODRÍGUEZ ${ }^{1,2}$, Rodrigo Hernando FALCÓN AYAPI ${ }^{1}$, \\ Rocío Esther DÍAZ VÁSQUEZ ${ }^{1}$
}

1 Instituto de Investigaciones de la Amazonía Peruana (IIAP). Av. Abelardo Quiñones km 2.5, Iquitos, Perú.mitza71@gmail.com

2 SOIL-PLANT SERVIS S.R.L. Calle Santa Rosa 546, Iquitos, Perú

\section{RESUMEN}

Consideramos que los cambios en la composición de especies de murciélagos ante impactos antrópicos, podrían evidenciar su potencial en función a especies indicadoras. Por ello, identificar estas especies en hábitats perturbados utilizando murciélagos se ha convertido en el propósito de este estudio. Aplicamos el método de redes de neblina durante 20 noches en dos tipos de hábitats de la Reserva Nacional Allpahuayo Mishana: Bosques perturbados, correspondientes a cultivos y Bosques naturales sin alteración. Durante cada noche, fueron instaladas 16 redes de neblina, activas desde las 18:00 a 24 horas y revisadas cada 30 minutos. Capturamos 451 individuos correspondientes a 37 especies aplicando un esfuerzo de $1936 \mathrm{~h} / \mathrm{red}$. Registramos especies indicadoras de bosques perturbados ( $\mathrm{p}=0.0003$ ), siendo la más destacable Sturnira tildae, seguido de Carollia perspicillata, Sturnira lilium, Carollia brevicauda y Carollia benkeithi. La especie más abundante en ambos tipos de bosques fue Artibeus planirostris y probablemente la más importante en los procesos de regeneración natural de los bosques estudiados. Según el gremio alimentario, la mayor riqueza y abundancia de murciélagos frugívoros se encuentra en bosques perturbados (0.001). Según los resultados, los murciélagos constituyen un buen grupo taxonómico para aplicarse en métodos de estados de conservación de biodiversidad en bosques amazónicos; lo cual resultaría atractivo y generaría un impacto de costo beneficio ecológico, económico, social y científico.

PALABRAS CLAVES: Cultivos, chiroptera, especies pioneras, Loreto, Sturnira tildae 


\title{
BATS INDICATORS OF DISTURBED HABITATS IN THE ALLPAHUAYO MISHANA NATIONAL RESERVE, PERUVIAN AMAZON
}

\begin{abstract}
We consider that the changes in the composition of bat species in the face of anthropic impacts, could show its potential in terms of indicator species. Therefore, identify these species in disturbed habitats using bats has become the purpose of this study. We applied the mist network method for 20 nights in two types of habitats in the Allpahuayo Mishana National Reserve: Disturbed forests, corresponding to crops and natural forests without alteration. During each night, 16 mist nets were installed, active from 18:00 to 24:00 hours and reviewed every 30 minutes. We captured 451 individuals that corresponded to 37 species applying an effort of $1936 \mathrm{~h} /$ net. We recorded species indicating disturbed forests $(\mathrm{p}=0.0003)$, the most noteworthy being Sturnira tildae, followed by Carollia perspicillata, Sturnira lilium, Carollia brevicauda and Carollia benkeithi. The most abundant species in both types of forests was Artibeus planirostris and probably the most important in the natural regeneration processes of the forests studied. According to the food guild, the greatest wealth and abundance of frugivorous bats is found in disturbed forests $(p=0.001)$. According to the results, the bats constitute a good taxonomic group to be applied in the method of states of conservation of biodiversity in Amazonian forests; which would be attractive and generate an impact of ecological, economic, social and scientific cost benefit.

KEYWORDS: Chiroptera, crops, Loreto, pioneer species, Sturnira tildae
\end{abstract}




\section{INTRODUCCIÓN}

Las sociedades humanas producen cambios constantes en los bosques naturales, requieren de espacios, alimentos y tecnología para satisfacer sus necesidades (Butchart et al., 2006). Estos cambios a su vez necesitan ser evaluados de forma precisa para que permitan tomar medidas correctivas oportunas (Carignan \& Villard, 2002; Butchart et al., 2006). Estos reemplazos pueden ser evaluados mediante medidas de riqueza, abundancia y estructura poblacional de especies (Roberger \& Angelstam, 2004; Rodrigues \& Brooks, 2007), las que pueden generar enormes gastos y requerir mucho esfuerzo. Es así que en la Séptima Reunión del Convenio sobre la Diversidad Biológica 2004, se propone a las especies indicadoras como una estrategia para evaluar el estado de la biodiversidad y el cumplimiento de metas de conservación (Balmford et al., 2005; Dobson, 2005) a bajo costo y con menor esfuerzo. Los murciélagos presentan características únicas que merecen ser destacadas y aprovechadas para un mejor entendimiento ecológico de ecosistemas naturales y perturbados, como por ejemplo: la diversidad de su alimento, la facilidad de identificación, el monitoreo poblacional y los cambios de composición en áreas perturbadas (Jones et al., 2009). Los murciélagos realizan los mayores procesos de dispersión de semillas de forma eficaz (Fleming \& Sosa, 1994; GalindoGonzáles et al., 2000; Aroca et al., 2016) y resultan cruciales en la dinámica de regeneración de bosque tropical. Sin embargo, se conoce poco las interacciones que ocurren entre los murciélagos y sus hábitats. Las características de estos mamíferos voladores nos impulsaron a entender la composición y dinámica en áreas que han sufrido cambios con la finalidad de identificar especies indicadoras de áreas perturbadas. El monitoreo de la calidad de un ecosistema resultaría atractivo, rentable y sostenible a partir de murciélagos indicadores del estado de conservación de biodiversidad.

En la Amazonía peruana existen diversos estudios sobre la diversidad, uso de hábitat y nuevas especies de murcielagos (Gorchov et al.; 1993, Hice et al., 2004; Díaz, 2011; Rengifo et al., 2013; Velazco, 2014; Ramos-Rodríguez et al., 2017); pero, aún dentro de este grupo, se carece de conocimientos que permitan evidenciarlos como especies indicadoras de la calidad del ecosistema. La Reserva Nacional Allpahuayo Mishana (RNAM) alberga una gran diversidad mamíferos (Hice \& Velazco, 2002; OversluijsVásquez, 2003), siendo muy representativos los murciélagos con 63 especies (Hice et al., 2004).

Dentro de esta reserva se encuentra el Centro de Investigación Allpahuayo (CIA), con bosques naturales y áreas de cultivo de especies nativas. El CIA tiene una muestra representativa de murciélagos, con 31 especies (López-Wong, 2002), la cual representa el $49.21 \%$ de especies de la RNAM, y el $17.03 \%$ de especies del Perú. Por ello, tuvimos como objetivo identificar especies de murciélagos que sirvan como indicadores de bosques perturbados en función a la composición de especies y gremios alimenticios en este sector de la Amazonía peruana. Estos datos serían de utilidad en la aplicación de programas de monitoreo del estado de conservación de ecosistemas.

\section{MATERIALES Y MÉTODOS}

\section{ÁREA DE ESTUDIO}

El estudio se realizó en el Centro de Investigaciones de la RNAM, ubicado en el $\mathrm{km} 26.8$ de la carretera Iquitos-Nauta (S $3^{\circ} 58^{\prime}$, W $73^{\circ} 25^{\prime}$ ) a $148 \mathrm{msnm}$, al suroeste de la ciudad de Iquitos, en la Provincia de Maynas, Región Loreto (Figura 1, Tabla 1).

El área de estudio tiene una precipitación media anual que oscila entre 196.9 a 297 mm; 


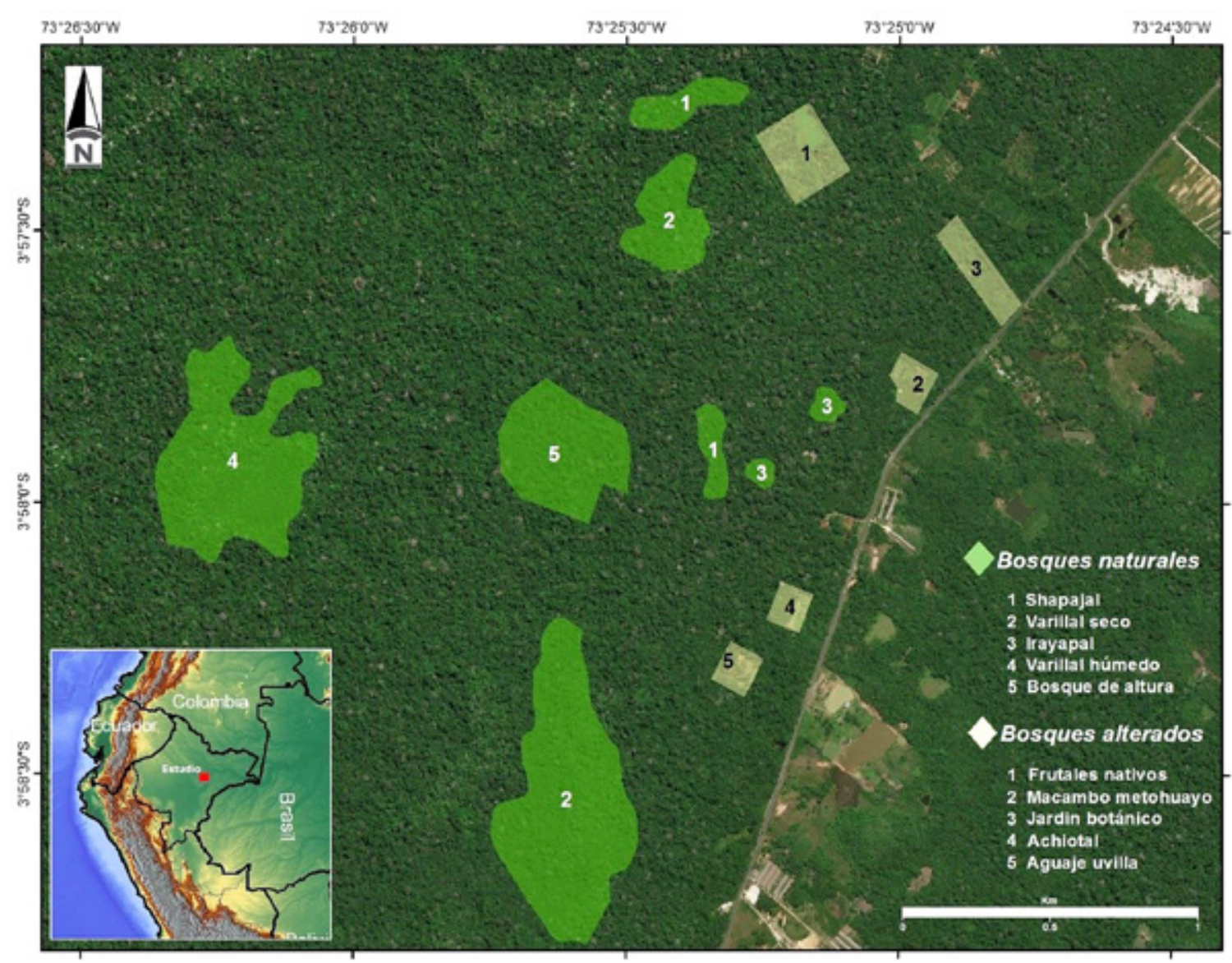

Figura 1. Mapa del área de estudio en el CIA, RNAM.

Tabla 1. Coordenadas geográficas de los bosques perturbados y naturales estudiados en el CIA, RNAM

\begin{tabular}{|c|c|c|c|}
\hline Código & Microhábitat & $\mathrm{X}$ & $\mathbf{Y}$ \\
\hline \multicolumn{4}{|c|}{ Bosques Perturbados } \\
\hline 1 & Frutales Nativos & -73.419572 & -3.955939 \\
\hline 2 & Macambo Metohuayo & -73.416146 & -3.963005 \\
\hline 3 & Aguaje Uvilla & -73.414361 & -3.959456 \\
\hline 4 & Achiote & -73.42005 & -3.969849 \\
\hline 5 & Jardín Botánico & -73.421929 & -3.971517 \\
\hline \multicolumn{4}{|c|}{ Bosques Naturales } \\
\hline \multirow{2}{*}{1} & \multirow{2}{*}{ Shapajal } & -73.423258 & -3.954391 \\
\hline & & -73.422400 & -3.965033 \\
\hline \multirow{2}{*}{2} & \multirow{2}{*}{ Varillal seco } & -73.423764 & -3.958000 \\
\hline & & -73.426855 & -3.975767 \\
\hline \multirow{2}{*}{3} & \multirow{2}{*}{ Irapayal } & -73.418918 & -3.96367 \\
\hline & & -73.420922 & -3.965736 \\
\hline 4 & Varillal húmedo & -73.437086 & -3.965396 \\
\hline 5 & Bosque de altura & -73.427253 & -3.965178 \\
\hline
\end{tabular}


con valores extremos mínimos en junio y agosto (113.8 y $87 \mathrm{~mm}$ ) y máximos en marzo y noviembre (503.7 y $519.4 \mathrm{~mm}$ ). La temperatura media anual oscila entre 27.2 a $29.4^{\circ} \mathrm{C}$. La humedad relativa es casi constante a lo largo del año, y es cercano al 85\% (Paredes, 2012).

Se evaluaron dos tipos de hábitats: 1 . Bosques perturbados; que corresponden a cultivos con más de 10 años de antigüedad, como: Aguaje Mauritia flexuosa, Uvilla Pourouma cecropiifolia, Macambo Theobroma bicolor, Metohuayo Caryodendron orinocense, Achiote Bixa orellana, frutales nativos y plantas medicinales. 2. Bosques naturales, que corresponden al bosque control, sin alteración antrópica, aquí tenemos bosques sobre suelo de arena blanca tipo seco y húmedo, bosque de Atalea racemosa "shapajal", bosques con dominancia de Lepidocaryum tenue "irapayal" y bosque con dosel muy alto.

Los bosques perturbados (cultivos) se caracterizaron por tener un buen drenaje de suelo, vegetación menor a $20 \mathrm{~m}$ de alto. Las especies de árboles representativos en el dosel fueron Apeiba glabra, Bactris gasipaes, Caryodendron orinocense, Cecropia sciadophylla, Dialium guianense, Euterpe oleracea, Jacaranda copaia, Mauritia flexuosa, Pourouma cecropiifolia. En el estrato medio fueron Caryodendron orinocense, Mangifera indica, Spondias mombin, Solanum sp., Tetrathylacium macrophyllum, Theobroma bicolor, Tococa guianensis, Vismia gracilis, Vismia macrophylla; y en el sotobosque a Calathea sp., Chelonanthus alatus, Clidemia hirta, Clitoria sp., Croton lecheri, Cyperus sp., Costus scaber, Cyclanthus bipartitus, Doliocarpus sp., Piper sp., Selaginella conduplicata. Entre las plantas medicinales estuvieron Uncaria tomentosa, Uncaria guianensis, Banisteriopsis caapi, Brumfelsia grandiflora, Maytenus macrocarpa y Mansoa alliacea, entre otros.

Los bosques naturales (bosques de control) se caracterizaron por tener un buen drenaje de 0-13 cm de materia orgánica y altura entre 15 a $35 \mathrm{~m}$. Las especies de árboles representativos en el dosel fueron Adiscanthus fusciflorus, Aspidosperma pichonianum, Attalea butyracea, Buchenavia reticulata, Chrysophyllum manaosensis, Eschweilera coriacea, Iryanthera laevis, Iryanthera paraensis, Nealchornea yapurensis, Oxandra euneura, Pouteria torta, Styrax sp. En el estrato medio se registra a Attalea butyracea, Attalea sp., Miconia sp., Protium divaricatum, Pseudosenefeldera inclinata, Virola pavonis; $y$ en el sotobosque a Cyclanthus bipartitus, Brosimum sp., Geonoma sp, Lepidocaryum tenue, Ischnosiphon sp., entre otros.

\section{CAPTURA Y DETERMINACIÓN DE ESPECIES DE MURCIÉLAGOS}

La captura de los murciélagos se realizó durante 20 días, entre los meses de agosto y setiembre de 2016. En cada tipo de hábitat se tuvieron dos réplicas, cada una conformada por 16 redes de neblina de $2.6 \times 12 \mathrm{~m}$ con $30 \mathrm{~mm}$ de apertura de malla. Las redes fueron ubicadas fuera de los senderos del área de estudio, permaneciendo desplegadas durante seis horas (18:00-24:00 horas) y revisadas cada hora.

Los murciélagos capturados se mantuvieron en bolsas de tela blanca durante 10 a 30 minutos, para suposterior identificación. Se registró el sexo, edad (mediante el grado de fusión de las epífisis metacarpales), estado reproductivo (machos con testículo escrotal o abdominal, hembras activas con vagina abierta o inactiva con vagina cerrada), también se tomó medidas morfométricas y masa corporal. La determinación del gremio alimentario se realizó por referencia de Gardner (1977) y por observación directa del contenido fecal de los murciélagos capturados. Además se colectaron muestras fecales con el propósito de registrar las especies de plantas consumidas (50\% del contenido fecal para germinación y el $50 \%$ para preservación), seguidamente 
los murciélagos fueron liberados in situ. La identificación taxonómica se realizó con Simmons (2006) y Gardner (2008) y la nomenclatura sigue a Solari \& Martínez-Arias (2014).

\section{ANÁLISIS DE DATOS}

La estimación de la riqueza esperada se realizó con los estimadores: Chao 1, Jacknife1, Jacknife2, Boopstrap y Michaelis y Menten, analizados con el programa SDR 4.1.2. (Seaby \& Henderson, 2007). La riqueza, dominancia y especies efectivas, obtenido del exponencial del índice de entropía de Shannon (Jost, 2006) fueron analizados en el programa Past 3.19 (Hammer et al., 2001).

La determinación de las especies más abundantes se realizó con la curva de Whittaker, donde se ordenó secuencialmente desde las especies más hasta las menos abundantes a lo largo del eje horizontal, con la finalidad de facilitar la comparación entre los diferentes hábitats (Magurran, 2004).

La identificación de las especies y los gremios alimenticios más importantes o con mayor variabilidad entre ambos tipos de hábitats fue realizada con el Análisis de Componentes

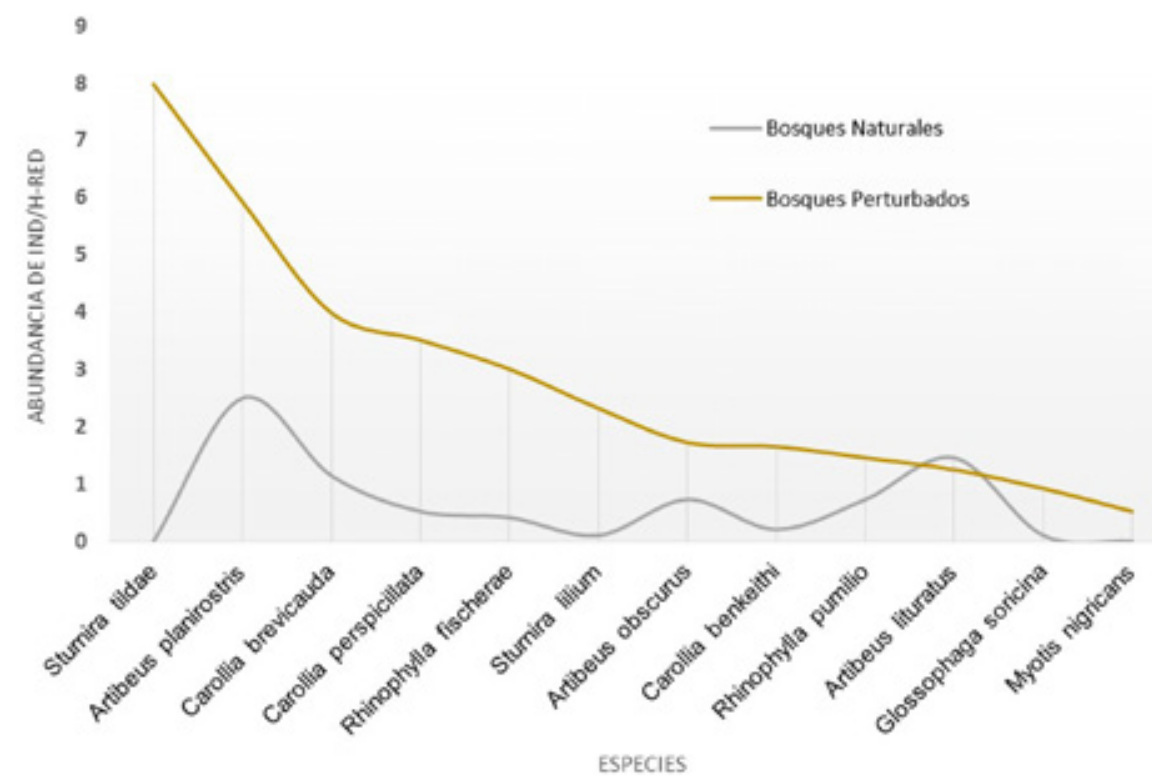

Figura 2. Curva de orden de especies de murciélagos en el CIA, RNAM.
Principales (ACP), donde los resultados necesitan al menos el 50\% de explicación de los componentes principales ( 1 o 2 axis) para ser considerados (Henderson \& Seaby, 2008). Para evaluar si los grupos previamente definidos son estadísticamente significativos aplicamos el Análisis de Similitud (ANOSIM), donde las muestras dentro de grupos deben de ser más similares en composición que muestras provenientes de diferentes grupos. El método usa el índice de Bray Curtis, con la hipótesis nula que no hay diferencias entre los miembros de varios grupos (Henderson \& Seaby, 2008). Ambos análisis fueron realizados en el software Community Analysis Package (CAP) 4.0.

\section{RESULTADOS}

Se capturó 451 individuos de 37 especies de murciélagos con un esfuerzo de $1936 \mathrm{~h} / \mathrm{red}$. La familia Phyllostomidae representó el 98.89\% de las capturas. Las especies más abundantes fueron Artibeus planirostris (17.96\%, 81 ind.) y Sturnira tildea (17.07\%, 77 ind.) (Figura 2, Tabla 2).

El análisis de componentes principales de la composición de especies por tipo de bosque indicó que la variabilidad puede ser explicada al $63.7 \%$ en dos componentes. El componente I explica el $44.4 \%$, en donde las especies Sturnira tildae, Carollia perspicillata, Sturnira lilium, Carollia brevicauda y Carollia benkeithi resultan potenciales indicadoras de bosque perturbados. El Componente II explicó el $19.3 \%$ y las especies más importantes fueron Artibeus lituratus y Rhynophyla 
Tabla 2. Composición de murciélagos por hábitat en la EBJAA en la RNAM

\begin{tabular}{|c|c|c|c|c|c|c|c|c|c|c|}
\hline \multirow[b]{2}{*}{ Especies } & \multicolumn{5}{|c|}{ Bosques naturales } & \multicolumn{5}{|c|}{ Bosques perturbados } \\
\hline & $\begin{array}{l}\text { Varillal } \\
\text { seco }\end{array}$ & $\begin{array}{l}\text { Bosque } \\
\text { Alto }\end{array}$ & Irapayal & Shapajal & $\begin{array}{l}\text { Varillal } \\
\text { húmedo }\end{array}$ & Achiotal & $\begin{array}{c}\text { Jardín } \\
\text { Botánico }\end{array}$ & Aguajal & $\begin{array}{l}\text { Frutales } \\
\text { nativos }\end{array}$ & $\begin{array}{l}\text { Macambo/ } \\
\text { Metohuayo }\end{array}$ \\
\hline Carollia benkeithi & 1 & 1 & 0 & 0 & 0 & 1 & 5 & 4 & 1 & 5 \\
\hline Carollia brevicauda & 6 & 2 & 1 & 0 & 2 & 8 & 4 & 7 & 7 & 13 \\
\hline Carollia perspicillata & 2 & 1 & 1 & 1 & 0 & 3 & 4 & 8 & 8 & 11 \\
\hline Rhinophylla fischerae & 3 & 0 & 0 & 0 & 1 & 1 & 27 & 1 & 0 & 0 \\
\hline Rhinophylla pumilio & 0 & 4 & 0 & 1 & 2 & 0 & 1 & 8 & 1 & 4 \\
\hline Desmodus rotundus & 0 & 0 & 0 & 0 & 0 & 3 & 0 & 0 & 0 & 0 \\
\hline Choeroniscus minor & 0 & 0 & 0 & 0 & 0 & 0 & 0 & 0 & 1 & 0 \\
\hline Glossophaga soricina & 0 & 0 & 0 & 1 & 0 & 2 & 0 & 0 & 3 & 4 \\
\hline Lonchophylla thomasi & 0 & 2 & 0 & 0 & 0 & 0 & 0 & 0 & 1 & 0 \\
\hline Chrotopterus auritus & 2 & 0 & 0 & 0 & 0 & 0 & 0 & 0 & 0 & 0 \\
\hline Phyllostomus astatus & 0 & 0 & 0 & 1 & 0 & 0 & 0 & 0 & 0 & 0 \\
\hline Phyllostomus elongatus & 0 & 0 & 1 & 0 & 0 & 0 & 0 & 0 & 0 & 0 \\
\hline Micronycteris minuta & 0 & 0 & 0 & 1 & 0 & 0 & 0 & 0 & 0 & 0 \\
\hline Tonatia bidens & 0 & 0 & 1 & 0 & 0 & 0 & 0 & 0 & 0 & 0 \\
\hline Tonatia saurophila & 0 & 0 & 1 & 1 & 0 & 0 & 0 & 0 & 0 & 0 \\
\hline Trachops cirrhosus & 0 & 0 & 0 & 1 & 0 & 0 & 0 & 0 & 0 & 0 \\
\hline Trinycteris nicefori & 0 & 1 & 0 & 0 & 0 & 0 & 0 & 0 & 0 & 0 \\
\hline Artibeus lituratus & 3 & 4 & 2 & 3 & 2 & 0 & 0 & 3 & 0 & 9 \\
\hline Artibeus obscurus & 1 & 1 & 1 & 2 & 2 & 5 & 6 & 1 & 4 & 1 \\
\hline Artibeus planirostris & 9 & 2 & 6 & 3 & 4 & 1 & 10 & 16 & 2 & 28 \\
\hline Chiroderma salvini & 0 & 0 & 0 & 0 & 0 & 0 & 1 & 0 & 0 & 0 \\
\hline Chiroderma trinitatum & 0 & 0 & 0 & 0 & 0 & 0 & 1 & 0 & 0 & 0 \\
\hline Chiroderma villosum & 0 & 0 & 0 & 0 & 0 & 1 & 0 & 0 & 0 & 0 \\
\hline Dermanura anderseni & 0 & 1 & 0 & 0 & 0 & 0 & 0 & 0 & 0 & 0 \\
\hline Dermanura gnoma & 0 & 0 & 0 & 0 & 0 & 0 & 0 & 0 & 1 & 0 \\
\hline Mesophylla macconnelli & 0 & 0 & 0 & 1 & 0 & 1 & 0 & 0 & 0 & 0 \\
\hline Platyrrhinus incarum & 0 & 0 & 0 & 0 & 0 & 1 & 0 & 0 & 0 & 0 \\
\hline Sturnira lilium & 0 & 0 & 0 & 1 & 0 & 5 & 3 & 3 & 4 & 7 \\
\hline Sturnira magna & 0 & 0 & 0 & 0 & 0 & 0 & 1 & 0 & 0 & 1 \\
\hline Sturnira sp. & 0 & 0 & 0 & 0 & 0 & 0 & 0 & 0 & 0 & 1 \\
\hline Sturnira tildae & 0 & 0 & 0 & 0 & 0 & 3 & 3 & 30 & 14 & 27 \\
\hline Uroderma bilobatum & 1 & 0 & 0 & 0 & 0 & 0 & 0 & 0 & 0 & 0 \\
\hline Vampiressa pusilla & 1 & 0 & 0 & 0 & 0 & 0 & 2 & 0 & 0 & 0 \\
\hline Vampyriscus bidens & 0 & 6 & 0 & 0 & 0 & 1 & 0 & 1 & 1 & 2 \\
\hline Vampyrodes caraccioli & 1 & 0 & 0 & 0 & 0 & 0 & 0 & 0 & 0 & 0 \\
\hline Myotis nigricans & 0 & 0 & 0 & 0 & 0 & 0 & 3 & 1 & 0 & 1 \\
\hline Individuos & 30 & 25 & 14 & 17 & 13 & 36 & 71 & 83 & 48 & 114 \\
\hline Promedio & & & 17 & & & & & 71 & & \\
\hline Especies & 11 & 11 & 8 & 12 & 6 & 14 & 14 & 12 & 13 & 14 \\
\hline Promedio & & & 11 & & & & & 14 & & \\
\hline
\end{tabular}



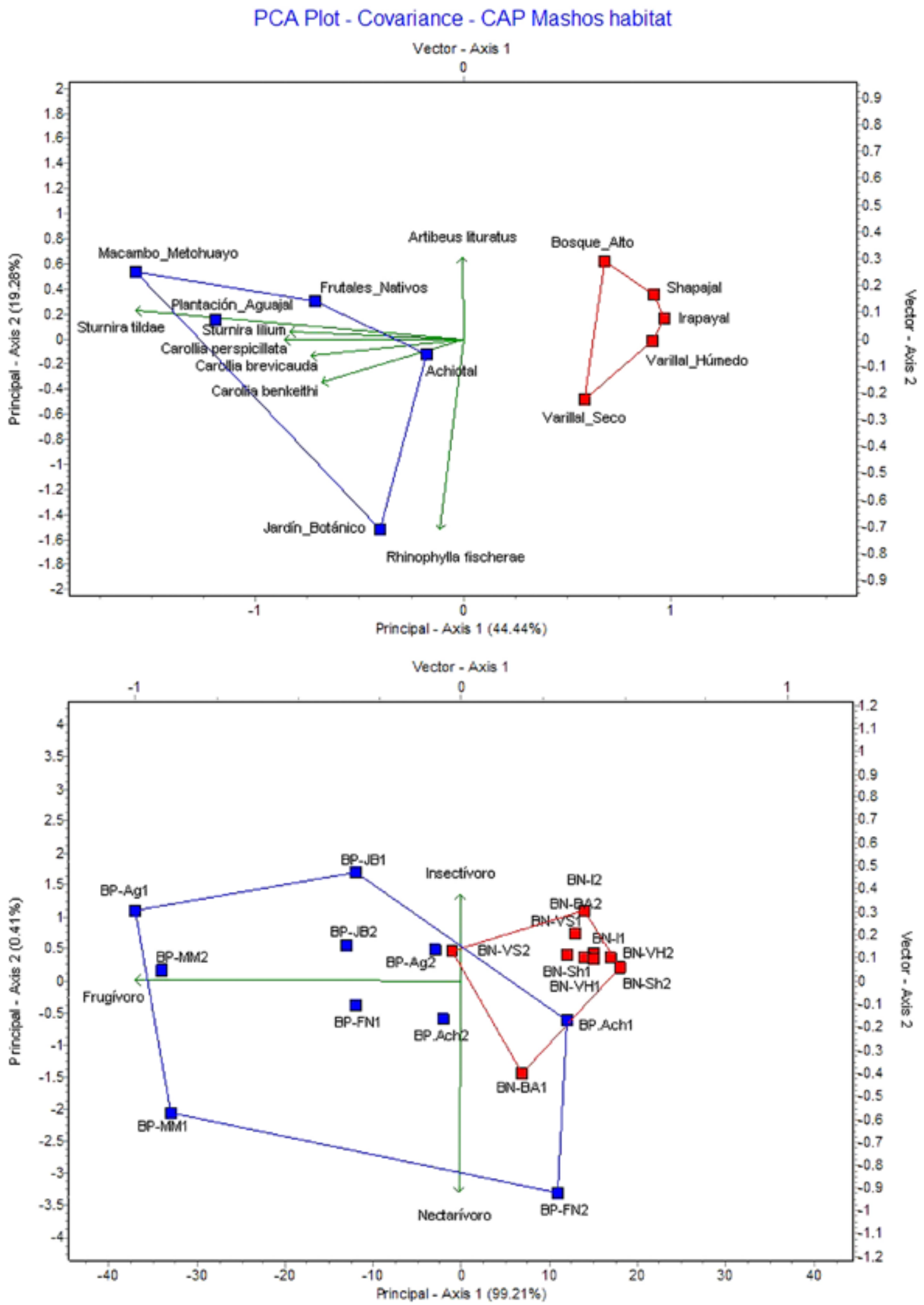

Figura 3. Análisis de Componentes Principales y ANOSIM. a). Murciélagos por tipo de bosque b). Gremios alimenticios por tipo de bosque de CIA, RNAM. 
fischerae, la primera estaba en áreas cultivadas con mayor cobertura arbórea, mientras que la última especie fue muy importante en las áreas cultivadas muy abiertas (Figura 3a).

El análisis de componentes principales de los gremios alimenticios por tipo de bosque, indicó que la variabilidad puede ser explicada al $99.62 \%$ en dos componentes con una significancia de 0.001. El componente I explica el 99.21\%, donde el gremio frugívoro es el potencial indicador de bosques perturbados. El Componente II explica el $0.42 \%$, donde los gremios insectívoros y nectarívoros representaron ambos tipos de bosques (Figura 3.b). En tanto, los gremios omnívoro, carnívoro, hematófago y nectarívoro no fueron representativos por las escasas capturas durante los muestreos.

Los bosques naturales y perturbados sólo comparten el $40 \%$ de especies de murciélagos. En bosques perturbados registramos 10 especies que no fueron registrados en bosques naturales (Chiroderma salvini, Chiroderma trinitatum, Chiroderma villosum, Choeroniscus minor, Dermanura gnoma, Desmodus rotundus, Myotis nigricans, Platyrrhinus incarum, Sturnira magna, Sturnira tildae). Mientras que en bosques naturales registramos 11 especies que no fueron registrados en bosques perturbados (Chrotopterus auritus, Dermanura anderseni, Micronycteris minuta, Phyllostomus elongatus, Phyllostomus hastatus, Tonatia bidens, Tonatia saurophila, Trachops cirrhosus, Trinycteris nicefori, Uroderma bilobatum, Vampyrodes caraccioli).

Los bosques naturales tuvieron mayor riqueza de especies que los bosques perturbados $(\mathrm{U}=3.402$, $\mathrm{P}=0.0007)$. La riqueza observada en los bosques perturbados representa el $75.76 \%$ (24 especies) de la riqueza esperada (30 especies). Mientras que la riqueza observada de los bosques naturales representa el 64.94\% (25 especies) de la riqueza estimada (39 especies). En tanto, los bosques naturales requieren de mayor esfuerzo para representar un mayor número de especies (Figura 4).
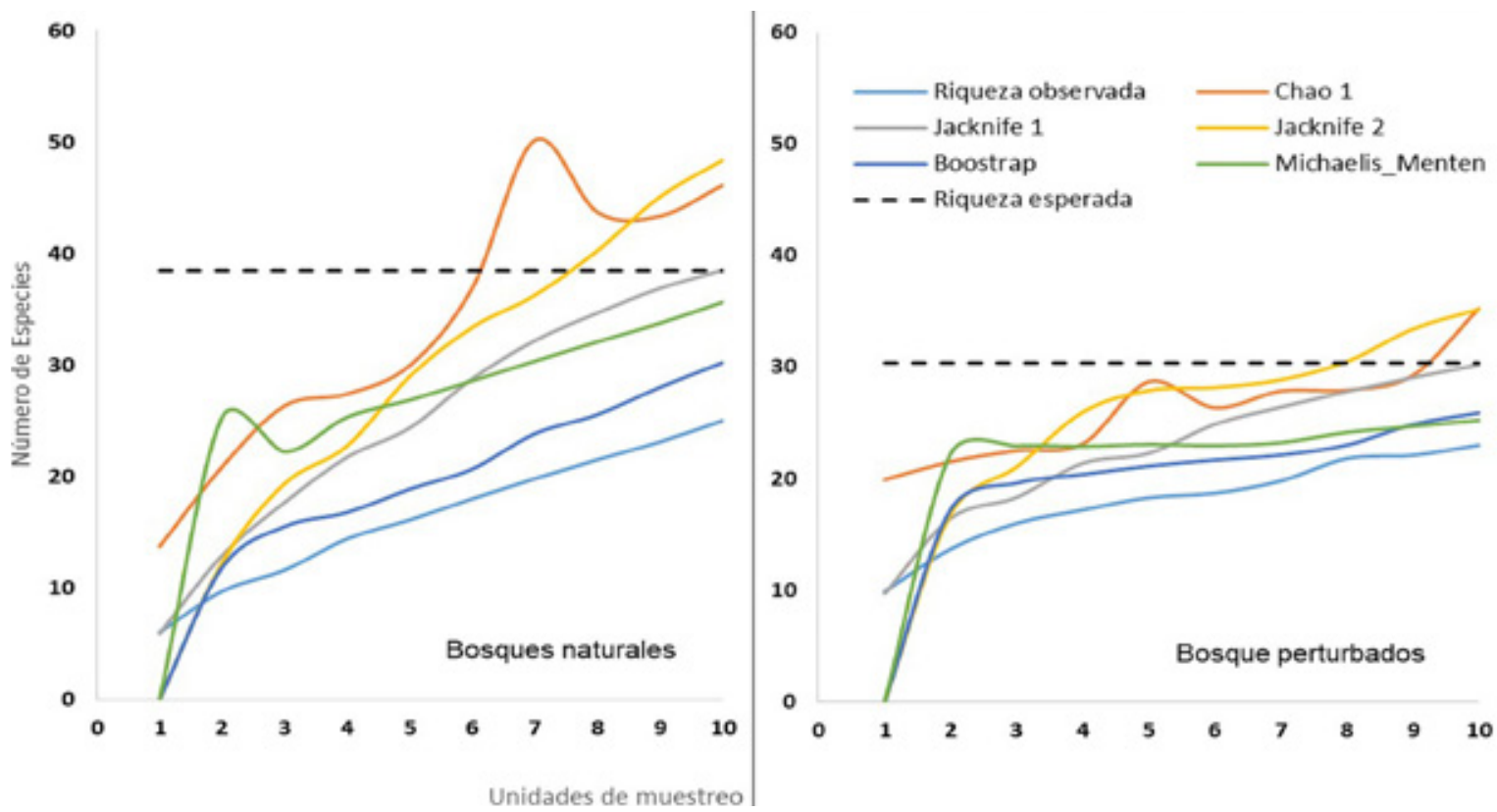

Figura 4. Riqueza estimada de especies de murciélagos por tipo de bosques naturales en el CIA, RNAM. 
La abundancia de los murciélagos difiere entre ambos bosques ( $\mathrm{t}$-student=-5.213, $\mathrm{P}<0.0001$ ). Los bosques perturbados presentan mayor abundancia (36.19 ind/h-red) que los bosques naturales (10.31 ind/h-red), mostrando una proporción de 3.5 a 1 individuos respectivamente. Las semillas contenidas en las heces de los murciélagos fueron identificadas a nivel de especie (12.9\%), a nivel de género (58.1\%) $\mathrm{y}$ hubo aquellos que no fueron identificados (29.0\%). Las semillas germinadas en laboratorio correspondieron a Cecropia sp. $1(\mathrm{n}=27)$, Ficus sp. $4(\mathrm{n}=1)$, Ficus sp. $5(\mathrm{n}=2)$, sp. $7(\mathrm{n}=2)$, sp. 8 $(\mathrm{n}=3)$ (Tabla 3). Las plantas consumidas con mayor frecuencia por los murciélagos fueron Piper sp. 1 (30.6\%), Cecropia sp. 1 (12.2\%) y
Ficus sp. 1 (7.1\%); mientras que las especies de murciélagos que consumieron mayor número de plantas fueron Carollia brevicauda, Sturnira tildae y Artibeus planirostris, especies que dominan los bosques perturbados (Figura 5).

\section{DISCUSIÓN}

En el estudio registramos una riqueza de 37 especies de murciélagos, correspondientes al $58.73 \%$ de murciélagos reportados para la RNAM (63 especies, Hice et al., 2004) y al $20.33 \%$ de especies de murciélagos del Perú (182 especies, Velazco, 2017); porcentajes considerables que resaltan el valor ecológico funcional de

Tabla 3. Frecuencia de contenido fecal con semillas de plantas consumidas por murciélagos en el Centro de Investigaciones Allapahuayo, Reserva Nacional Allpahuayo Mishana.

\begin{tabular}{|c|c|c|c|c|c|c|c|c|c|c|c|c|c|c|c|c|c|c|c|c|c|c|c|c|c|c|c|c|c|c|c|c|}
\hline & Especie de Plantas & $\begin{array}{c}-1 \\
\dot{2} \\
\hat{n} \\
\bar{d} \\
\frac{2}{2} \\
\frac{2}{2}\end{array}$ & 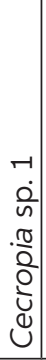 & 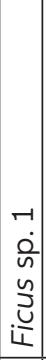 & $\begin{array}{l}N \\
0 \\
0 \\
0 \\
\frac{1}{0} \\
\frac{1}{0} \\
\frac{1}{d} \\
\frac{d}{0} \\
\frac{0}{2} \\
\frac{1}{2} \\
\end{array}$ & $\begin{array}{l} \\
n \\
\dot{0} \\
\dot{0} \\
\bar{\emptyset} \\
\frac{2}{2}\end{array}$ & 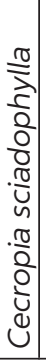 & 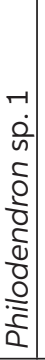 & 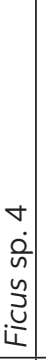 & 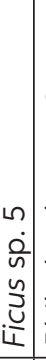 & 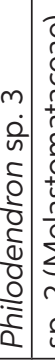 & 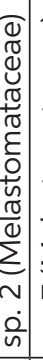 & 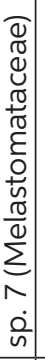 & 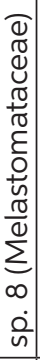 & 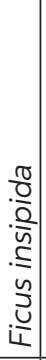 & 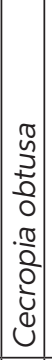 & 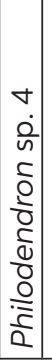 & $\begin{array}{c} \\
\dot{0} \\
\frac{0}{0} \\
\dot{0} \\
\frac{5}{3} \\
\frac{0}{0} \\
\frac{0}{\square}\end{array}$ & 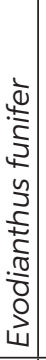 & 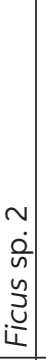 & 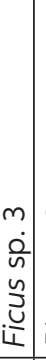 & $\begin{array}{l}v \\
\dot{2} \\
\stackrel{0}{n} \\
\bar{d} \\
\frac{2}{2} \\
\frac{2}{2}\end{array}$ & 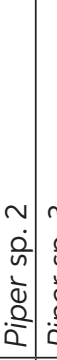 & 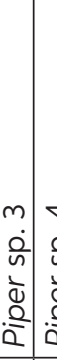 & 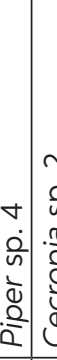 & 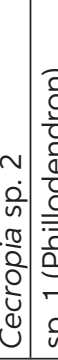 & 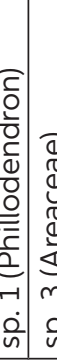 & 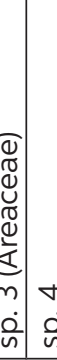 & & 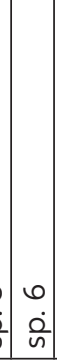 & 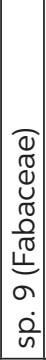 & 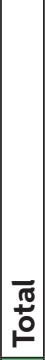 \\
\hline \multirow{16}{*}{ 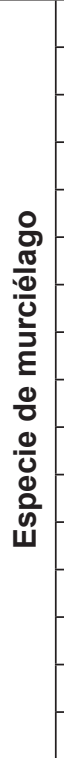 } & Carollia brevicauda & 8 & 1 & & & & & 1 & 1 & & & & & 1 & 1 & & & & & & & 1 & & 1 & & & 11 & 11 & & & & 18 \\
\hline & Sturnira tildae & 8 & 1 & 1 & 2 & 3 & & & & & & & & & & & 1 & 1 & & & & & 1 & & & & & & & & & 18 \\
\hline & Artibeus planirostris & 2 & 4 & 2 & 1 & & 1 & & & 2 & & & & & & & & & & & 1 & & & & & & & & & & & 13 \\
\hline & Carollia perspicillata & 4 & & & & & & & 1 & & & 1 & 1 & 1 & & & & & & & & & & & 1 & & & & & & 1 & 10 \\
\hline & Carollia benkeithi & 4 & & & & 1 & 1 & & & & & & 1 & & & & & & & 1 & & & & & & & & & & & & 8 \\
\hline & Rhinophylla pumilio & 2 & 1 & & & & & 1 & & & & & & & & & & & & & & & & & & & & & & 1 & & 5 \\
\hline & Sturnira lilium & 2 & & & & & & 1 & & & 11 & 1 & & & & 1 & & & & & & & & & & & & & & & & 6 \\
\hline & Artibeus lituratus & & 2 & & & & 2 & & & & & & & & & & & & & & & & & & & & & & & & & 4 \\
\hline & Artibeus obscurus & & 1 & & & & & & & 1 & & & & & & & & & & & & & & & & 1 & & & & & & 3 \\
\hline & Rhinophylla fischerae & & & & 1 & & & & 1 & & & & & & & & & & 1 & & & & & & & & & & & & & 3 \\
\hline & Vampyriscus bidens & & & 2 & & & & & & & & & & & & 1 & & & & & & & & & & & & & & & & 3 \\
\hline & Chiroderma salvini & & & 1 & & & & & & & & & & & & & & & & & & & & & & & & & 1 & & & 2 \\
\hline & Glossophaga soricina & & 1 & & & & & & & & 1 & & & & & & & & & & & & & & & & & & & & & 2 \\
\hline & Dermanura anderseni & & & 1 & & & & & & & & & & & & & & & & & & & & & & & & & & & & 1 \\
\hline & Platyrrhinus incarum & & 1 & & & & & & & & & & & & & & & & & & & & & & & & & & & & & 1 \\
\hline & Vampiressa pusilla & & & & & & & & & & & & & & 1 & & & & & & & & & & & & & & & & & 1 \\
\hline & Total & 30 & 12 & 7 & 4 & 4 & 4 & 3 & 3 & 3 & 2 & 2 & 2 & 2 & 2 & 2 & 1 & 1 & 1 & 1 & 1 & 1 & 1 & $1 \mid 1$ & 11 & $1 \mid 1$ & $1 \quad 1$ & $1 \mid 1$ & 1 & 1 & 1 & \\
\hline
\end{tabular}



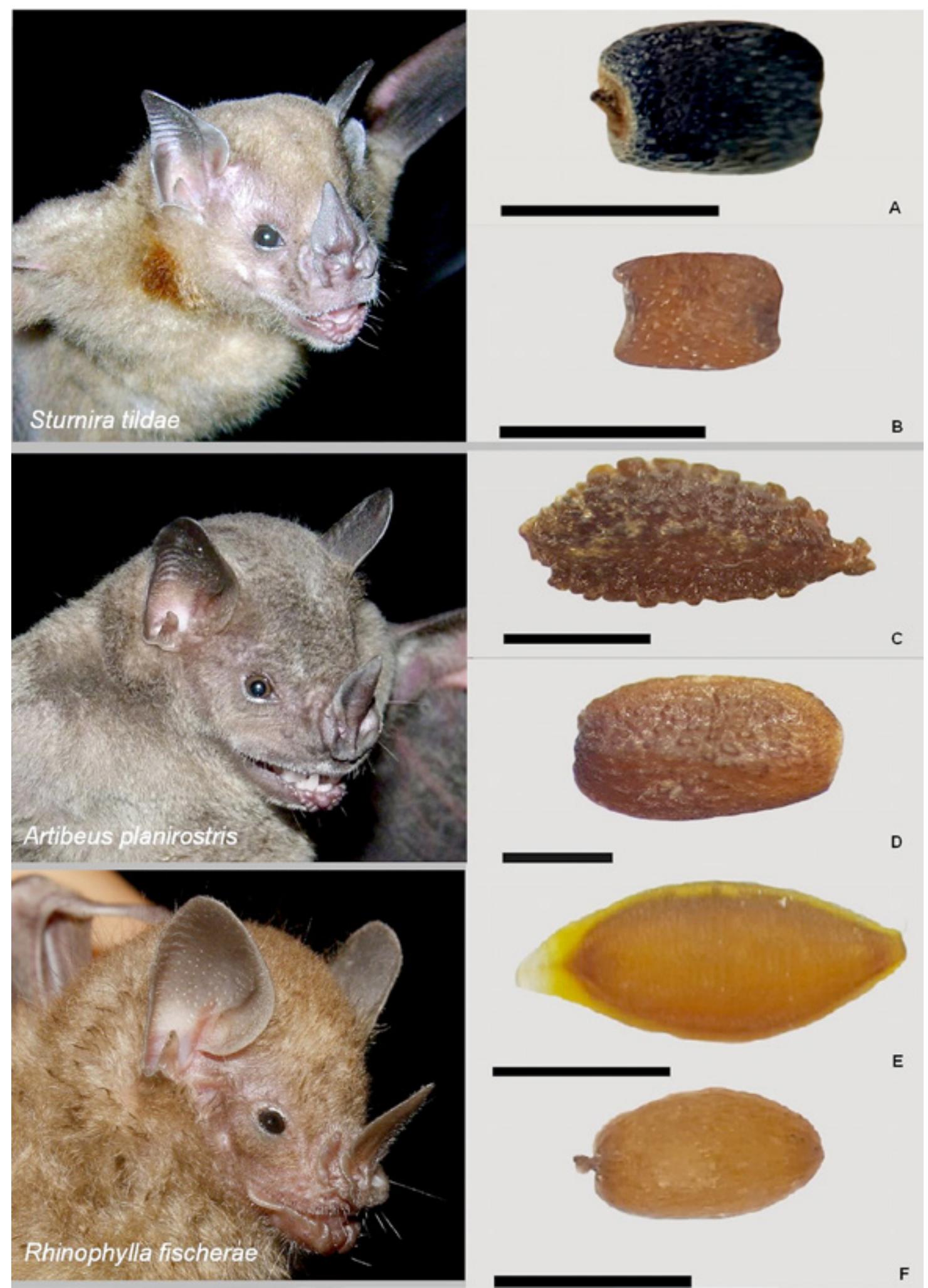

Figura 5. Murciélagos frugívoros y semillas consumidas en el CIA, RNAM. A. Piper sp. 1 B. Piper sp. 5 C. Cecropia sciadophylla D. Cecropia sp. 1 E. Evodiantus funifer F. Philodendron sp. 2. Escala $1 \mathrm{~mm}$. 
las especies habitantes del área de estudio. $A$. planirostris, fue registrado en abundancia en ambos tipos de bosques. Oliveira \& Lemes (2010 y Reis et al. (2007) mencionan que A. planirostris es un excelente dispersador de semillas, principalmente de plantas pioneras encontradas en la fase inicial de sucesión, convirtiéndose en una especie crucial para bosques perturbados y naturales. Así también, Ríos-Blanco (2010), evidenció que en parches de bosques secos tropicales de Colombia abunda A. planirostris consumiendo frutos de Ficus, Vismia y Cecropia. En nuestro estudio, la amplitud alimentaria de A. planirostris en función a especies heliófitas y esciófitas permitió registrarlo sin exclusividad de hábitats, por ello, no podríamos destacarlo como especie indicadora de hábitat; caso contrario lo sucedido con Sturnira tildae, que para fines de nuestro estudio, podríamos destacarla como especie indicadora de bosques perturbados. Sturnira tildae fue registrado con un abundancia de 7.98 ind./h-red y exclusivo de bosques perturbados, alimentándose de Piper, Cecropia, Ficus y Phyllodendron; inferimos que la disponibilidad de su alimento en bosques perturbados influye en la restricción espacial de su hábitat.

Los mayores conocimientos de género Sturnira, corresponden a Sturnira lilium. Prefieren en su dieta especies pioneras y en general especies heliófitas; es dispersor legítimo y efectivo de Solanaceae, (Mello et al., 2008; Passos et al. 2003), además dispersa Piper y Ficus (Acora et al., 2016). En bosques montanos los patrones de su dieta son similares. En los andes de Argentina las especies de Sturnira también prefieren Piper y Solanum (Giannini, 1999). La preferencia por estas especies de plantas permite destacar un tipo de mutualismo de dispersión-alimentación entre la planta y el murciélago respectivamente. La composición comunitaria de los murciélagos por tipo de bosque difiere significativamente
$(\mathrm{P}=0.003)$ debido a las especies Sturnira tildae, Carollia perspicillata, Sturnira lilium, Carollia brevicauday Carolliabenkeithi; especiesfrugívoras que abundaron los bosques perturbados y fueron consumidores exclusivos de especies heliófitas, factor principal que explicaría la divergencia comunitaria en los hábitats evaluados. Jones et al. (2009), refiere que las alteraciones de hábitats naturales influencian en la diversidad y abundancia de murciélagos brindándoles el valor de bioindicadores. En nuestro estudio observamos riquezas similares y abundancias diferentes por tipo de hábitat.

Al estudiar la variabilidad de los gremios alimentarios por tipo de bosque, observamos que los murciélagos frugívoros de bosques perturbados se diferencian de los otros gremios. Registramos 19 especies con 333 individuos de murciélagos frugívoros en bosques perturbados y 15 especies con 86 individuos en los bosques naturales. La distribución natural de murciélagos frugívoros en bosques perturbados podría ser un indicador de tipo de bosque. Sería necesario implementar un estudio que permita estimar la abundancia de frugívoros según el tipo y tiempo de perturbación.

Entre los otros gremios alimentarios, los insectívoros fueron más diversos en bosques naturales, sin embargo Myotis nigricans fue la única especie capturada en bosques perturbados en cinco oportunidades; según Wilson (1971), este comportamiento está influenciado por la mayor disponibilidad de su alimento entre 0 a $10 \mathrm{~m}$ de alto, teniendo mayor éxito en áreas de segundo crecimiento; esto podría explicar las capturas exclusivas de Myotis nigricans en bosques perturbados. Morton (1989) menciona que los murciélagos insectívoros tienen un sistema de ecolocación bien agudo para capturar sus presas en el aire por lo que podrían detectar con facilidad las redes de neblina en áreas despejadas, además menciona que la mayoría 
prefieren los estratos medio y dosel del bosque. Además, Sánchez-Merlos et al. (2005) y Janzen (1991) evidencian que las mayores densidades de especies insectívoras corresponden a temporadas lluviosas donde prolifera su alimento. Los murciélagos insectívoros tienden a ser más susceptibles ante cambios en su hábitat y prefieren bosques naturales sin alteración pudiendo destacarse como especies indicadores los hábitats evaluados. Un estudio más profundo aplicando el método de redes de neblina y el acústico, permitiría conocer si él método aplicado influye en el registro de la riqueza y abundancia de especies insectívoras por hábitats. Laval \& Rodríguez (2002), mencionan que las especies del gremio carnívoro, ocupan el nivel más alto de la pirámide trófica, siendo los menos comunes y los más afectados frente a alteraciones de su hábitat, lo cual permiten destacarlo como especies indicadoras de hábitats naturales sin alteración.

Las especies frugívoras fueron las más abundantes en los bosques estudiados (62.9\%); según Fleming (1986) la abundancia de especies frugívoras en un determinado hábitat está influenciada por varios factores, entre ellos el alimento; la dietabásica delos murciélagosimplica una compensación entre la calidad y densidad del fruto. Los murciélagos que se alimentan de frutos de alta calidad como Piper y Solanum tienen dietas más amplias que aquellos que comen frutas de baja calidad pero de alta densidad como los Ficus. En nuestro estudio, los murciélagos frugívoros más abundantes compensaron sus dietas de calidad y densidad, con Piper, Ficus, Asplundia, Cecropia, entre otros, lo cual podría influenciar en un mejor establecimiento de las especies en los hábitats estudiados. Otros estudios muestran patrones diferentes, como los de Cosson et al. (1999), quienes capturaron escasos murciélagos frugívoros en áreas de cultivos de 0 a 1 año de antigüedad en la Guayana Francesa, atribuyendo a factores como rango domiciliario y estrategias de búsqueda de alimento los que influenciaron en sus resultados; probablemente la divergencia con nuestro estudio se debe al tiempo de antigüedad de los cultivos que pudo influenciar en la composición de especies visitantes de bosques perturbados.

Las Sub Familias más abundantes en el área de estudio fueron Stenodermatinae, Rhinophyllinae y Carolliinae. Sánchez-Merlos et al. (2005) mencionan que la abundancia de especies de estas sub familias se debe a la fácil adaptación por áreas perturbadas y dietas amplias ya que son especies con requerimientos generalizados de hábitat. En el estudio, Sturnira tildae, fue más abundante en bosques perturbados mientras que Artibeus planirostris representó los bosques naturales; ambas especies pertenecen a la sub familia Stenodermatinae, al gremio frugívoro y compartieron el $36.36 \%$ de su dieta en base a frutos de especies de plantas heliófitas como Cecropia sp. 1, Ficus sp. 1 y Philodendron sp. 2.

El 40\% de especies de murciélagos comparten ambas áreas de estudio, habitando ambas áreas indistintamente a factor de alteración; sin embargo las abundancias de estas especies difieren. El 60\% restante corresponde a únicas en su hábitat. De este porcentaje, el $28.6 \%$ de especies que fueron capturados en bosques perturbados pertenecen a los gremios frugívoros, nectarívoros y hematófagos; mientras que el $31.4 \%$ a los gremios insectívoros, omnívoros y carnívoros.

En definitiva, la regeneración de bosques perturbados como los cultivos dependen fundamentalmente del arduo trabajo realizado por los murciélagos frugívoros, ahí destacamos la importancia para su estudio y aplicación en procesos de regeneración y monitoreo de estados de conservación de biodiversidad de ecosistemas amazónicos. 


\section{CONCLUSIONES}

Las especies Sturnira tildae, Carollia perspicillata, Sturnira lilium, Carollia brevicauda y Carollia benkeithi son potenciales indicadoras de hábitats perturbados, la primera especie puede ser en mayor intensidad. La riqueza y abundancia de murciélagos del gremio frugívoro son potenciales indicadores de bosques perturbados, quienes consumieron frutos de Asplundia, Pipers, Philodendron, Cecropia y Ficus. En tanto, Artibeus planirostris puede ser la especie más importante como regeneradora de bosques naturales y perturbados, fue abundante en ambos hábitats y fue uno de los que consumió mayor número de especies de plantas.

\section{AGRADECIMIENTOS}

A Pedro Pérez y Fredy Arévalo por canalizar el financiamiento de la fase de campo y estadía en el Centro de Investigaciones Allpahuayo. A Maicon Hidalgo, Sixto Mananita y Jorge Ruíz, por el trabajo de campo durante los muestreos, así como a los practicantes y voluntarios de la Universidad de la Amazonía Peruana que nos acompañaron en el estudio. A Ricardo Zárate por la descripción del área de estudio e identificación de muestras de plantas consumidas por los murciélagos.

\section{BIBLIOGRAFÍA CITADA}

Aroca, A.K.; Hurtado, M.A.; Murillo-García, O.E. 2016. Diet Preference in Frugivorous Bats (Phyllostomidae) within a Fragment of Dry Tropical Forest. Revista de Ciencias, 20 (SPE):139- 146.

Álvarez, J.; Díaz, J.; Shany, N. 2012. Avifauna de la Reserva Nacional Allpahuayo Mishana, Loreto. Perú. Cotinga, 34: 61-84.
Balmford, A.; Bennun, L.; Ten Brink, B.; Cooper, D.; Côté, I.M.; Crane, P.; et al. 2005. The convention on biological diversity's 2010 target. Science, 307(5707): 212-213.

Butchart, S.H.; Akcakaya, H.R.; Kennedy, E.; HiltonTaylor, C. 2006. Biodiversity indicators based on trends in conservation status: strengths of the IUCN Red List Index. Conservation Biology, 20(2): 579-581.

Carignan, V.; Villard, M.A. 2002. Selecting indicator species to monitor ecological integrity: a review. Environmental monitoring and assessment, 78(1): 45-61.

Cosson, J.F.; Pons, J.M.; Masson, D. 1999. Effects of forest fragmentation on frugivorous and nectarivorous bats in French Guiana. Journal of Tropical Ecology, 15(4): 515-534.

Díaz, M. 2011. New records of bats from the northern region of the Peruvian Amazon. Zoological Research, 32:168-178.

Dobson, A. 2005. Monitoring global rates of biodiversity change: challenges that arise in meeting the Convention on Biological Diversity (CBD) 2010 goals. Philosophical Transactions of the Royal Society of London B: Biological Sciences, 360(1454): 229-241.

Fleming, T.H. 1986. Opportunism versus specialization: the evolution of feeding strategies in frugivorous bats. In Frugivores and seed dispersal (pp. 105-118). Springer, Dordrecht.

Fleming, T.H.; Sosa, V.J. 1994. Effects of nectarivorous and frugivorous mammals on reproductive success of plants. Journal of Mammalogy, 75:845-851.

Galindo-Gonzáles, J.; Guevara, S.; Sosa, V.J. 2000. Bat and bird-generated seed rains at isolated trees in pastures in a tropical rainforest. Conservation Biology, 14:1693- 1703.

Gardner, A.L. 1977. Feeding habits. In: Baker, R.J.; Jones, J. K.; Carter, D.C. (Eds). Biology of bats of the New World family Phyllostomatidae. Part 
II. Special Publication No. 13. The Museum, Texas Tech University, Lubbock. p. 293- 350.

Gardner, A.L. (Ed.). 2008. Mammals of South America, volume 1: marsupials, xenarthrans, shrews, and bats (Vol. 1). University of Chicago Press. p. 187 - 481.

Giannini, N. P. 1999. Selection of diet and elevation by sympatric species of Sturnira in an Andean rainforest. Journal of Mammalogy, 80(4): 1186- 1195.

Gorchov, D.L.; Cornejo, F.; Ascorra, C.; Jaramillo, M. 1993. The role of seed dispersal in the natural regeneration of rain forest after strip-cutting in the Peruvian Amazon. Vegetatio, 107(1): 339349.

Hammer, Ø.; Harper, D.A.T.; Ryan, P.D. 2001. PASTPalaeontological Statistics.sofware packege for education and data analysis. Paleontología Electrónica 4(1): 9pp.

Henderson P. y R. Seaby. 2008. A practical handbook for multivariate methods. Pisces Conservation. 224pp.

Hice, C.L.; Velazco, P.M. 2012. The non-volant mammals of the Reserva Nacional AllpahuayoMishana, Loreto, Perú. Special Publication of Texas Tech University 60:1-135.

Hice, C.L.; Velazco, P.M.; Willig, M.R. 2004. Bats of the Reserva Nacional Allpahuayo-Mishana, northeastern Peru, with notes on community structure. Acta Chiropterologica, 6(2): 319334.

Janzen, D.H. 1991. Historia Natural de Costa Rica. 1a ed. Editorial de la UCR, San José, Costa Rica. $822 \mathrm{pp}$.

Jones, G.; Jacobs, D.S.; Kunz, T.H.; Willig, M.R.; Racey, P.A. 2009. Carpe noctem: The importance of bats as bioindicators. Endangered species research, 8(1-2): 93-115.

Jost, L. 2006. Entropy and diversity. Oikos, 113(2): 363-375.

Laval, R.; Rodríguez, B. 2002. Murciélagos de Costa Rica, Santo Domingo de Heredia, Costa
Rica. Editorial del Instituto Nacional de Biodversidad (INBio), 320pp.

López-Wong, C. 2002. Uso de hábitat por quirópteros en la Zona Reservada AllpahuayoMishana. Tesis de pre-grado. Universidad Nacional de la Amazonia Peruana. Facultad de Ciencias Biológicas. Perú. 86 pp.

Magurran, A. 2004. Measuring Biological Diversity. Blackwell Science Ltd. 132pp.

Mello, M.A.R.; Kalko, E.K.V.; Silva, W.R. 2008. Movements of the bat Sturnira lilium and its role as a seed disperser of Solanaceae in the Brazilian Atlantic forest. Journal of Tropical Ecology, 24(2): 225- 228.

Morton, P.A.; Tuttle, M.D. 1989. Murciélagos tropicales americanos. Publicado por El Fondo Mundial para la Naturaleza, E.U.A. \& World Wildlife Fund.48pp.

Oliveira, A.K.M.; Lemes, F.T.F. 2010. Artibeus planirostris como dispersor e indutor de germinação em uma área do Pantanal do Negro, Mato Grosso do Sul, Brasil. Revista Brasileira de Biociências, 8(1).

Oversluijs-Vásquez, M. R. 2003. Animales de caza en la zona reservada AllpahuayoMishana. Folia Amazónica, 14(1), 7-16.

Paredes, M. 2012. Clima, Documento temático. Proyecto Microzonificación Ecológica y Económica del Área de Influencia de la Carretera Iquitos-Nauta, convenio entre el IIAP y DEVIDA. Iquitos, Perú. 88 pp.

Ramos-Rodríguez, M.C.; Cevillano, S.C.; Aquino, R.; Zárate, R.; Tirado, E.R. 2017. Diversidad de murciélagos en bosques de colina del río Itaya, Loreto, Perú. Folia Amazónica, 26(2).

Reis, N.R.; Peracchi, A.; Pedro, W.A., Lima, I.P. (Eds). 2007. Morcegos do Brasil. Londrina: Nélio R. dos Reis. 253pp.

Rengifo, E.M.; Calderón, W.; Aquino, R. 2013. Características de refugios de algunas especies de murciélagos en la cuenca alta del rio Itaya, Loreto, Perú. UNED Research Journal, 5(1). 
Ríos-Blanco, M.C. 2010. Dieta y dispersión efectiva de semillas por murciélagos frugívoros en un fragmento de bosque seco tropical, Córdoba, Colombia. Tesis de pre-grado. Pontificia Universidad Javeriana. Departamento de Biología. Bogotá, Colombia. 39 pp.

Roberger, J.M.; Angelstam, P.E.R. 2004. Usefulness of the umbrella species concept as a conservation tool. Conservation biology, 18(1): 76-85.

Rodrigues, A.S.; Brooks, T.M. 2007. Shortcuts for biodiversity conservation planning: the effectiveness of surrogates. Annu. Rev. Ecol. Evol. Syst., 38: 713-737.

Sánchez-Merlos, D.; Harvey, C.A.; Grijalva, A.; Medina, A.; Vílchez, S.; Hernández, B. 2005. Diversidad, composición y estructura de la vegetación en un agropaisaje ganadero en Matiguás, Nicaragua. Revista de Biologia Tropical, 53(3-4): 387-414.

Seaby, R.M.H.; Henderson P.A. 2007. Species diversity and richness version 4.1.2. PISCES Conservation Limited. (http://www. piscesconservation.com/freestuff .html). Acceso: 15/06/2018.
Simmons, N.B. 2006. Order Chiroptera. In Wilson, D.E.; Reeder, D.M. eds. Mammal species of the world: A taxonomic and geographic reference. 3rd ed. Smithsonian Institution Press, Washington, DC. p 312- 529.

Solari, S.; Martínez-Arias, V. 2014. Cambios recientes en la sistemática y taxonomía de murciélagos Neotropicales (Mammalia: Chiroptera). Therya, 5(1): 167-196.

Velazco, P.M., Gregorin, R.; Voss, R.S.; Simmons, N.B. 2014. Extraordinary local diversity of diskwinged bats (Thyropteridae: Thyroptera) in northeastern Peru, with the description of a new species and comments on roosting behavior. American Museum Novitates, 3795: 1-28.

Velazco, 2017. Lista actualizada de murciélagos del Peru. (http://www.paulvelazco.com/ murcielagos_peru.html). Acceso: 03/03/2018. Wilson, D.E. 1971. Ecology of Myotis nigricans (Mammalia: Chiroptera) on Barro Colorado Island, Panama Canal Zone. Journal of Zoology, 163(1), 1-13.

Recibido: 14 de marzo de 2018 Aceptado para publicación: 12 de julio de 2018 